\title{
Study on the Effect and Improvement of Responsibility Service of Rural Family Doctors in China
}

\author{
Lili Jia \\ School of Humanities and Management of Shaanxi University of Chinese Medicine, Xi'an, 712046
}

Keywords: rural family doctors; responsibility service; effect and improvement

\begin{abstract}
To change the pattern of rural health services and guide people to make full use of rural health services, since 2013, the Rural Health Service Center of Luzhou Road, Heyang, Hefei City has fully implemented the "Domestic Physician Responsibility System Service" with rural area as a carrier, family as a unit, prevention as a guide, and health as a goal, and vigorously publicized (knock step) signing services (specific measures), strict institutional arrangements (key), and fulfilling services (fundamental). After the implementation of the responsibility system of family doctors, residents' awareness of hypertension, diabetes, treatment rate, control rate, utilization rate of rural health services, satisfaction rate, etc., were significantly higher than before, and all had statistical significance. The implementation of the responsibility system for family doctors can enable rural residents to enjoy economic, convenient and high-quality medical and health care services, and improve the quality of life of patients with chronic diseases. The family doctor accountability system meets the needs of the government and rural residents for the development of rural health services.
\end{abstract}

\section{Introduction}

The grading diagnosis and treatment system is the key path for realizing the policy goal of the reform of the medical and health system, and is also the core content of the healthy Chinese strategy. The primary health organization "acceptable" is an important basis for achieving grading diagnosis and treatment. The breakthrough is to implement family doctors in line with China's national conditions. Signing service [1]. The family doctor signing service is the key to strengthening the function of the primary health care service network and realizing the grading diagnosis and treatment. It is also an important way to maintain the health of the people under the new situation.

\section{Objects and Methods}

There are altogether 20 village/neighborhood committees in the area of Datu Rural Health Service Center in Pudong New Area, Shanghai. As of December 2014, there were 58,661 residents. Using a convenient sampling method, 1,100 contracted residents of 20 villages/neighborhoods within the jurisdiction area were selected as the survey objects. Inclusion criteria: (1) Service time for contracted family doctors' responsibility system $\geq 1$ year; (2) Age $\geq 18$ years; (3) Basic literacy skills for normal communication; (4) Voluntary participation in the study. Exclusion criteria: (1) In the acute stage of major disease; (2) Cognitive impairment. 1. 2 Survey Tools A self-designed Survey Questionnaire for Family Doctors Responsibilities of Rural Health Service Centers was designed to conduct surveys on contracted residents. The questionnaire consists of 14 items, including: (1) Family doctors' ability to solve basic medical service problems in rural areas, including 2 items. They are: 1 Whether contracted residents are fixed to contract doctors; 2 Whether contracted family doctors can solve problems Basic medical service in rural areas. (2) The evaluation of the effectiveness of the family doctor's responsibility system includes 11 items. The contents of the services are as follows: 1 To carry out rural medical treatment, to sit in the village clinic (a rural clinic) for half a day; 2 To conduct an appointment for outpatient service, half a week Time visit to the clinic center (reservation clinic) 3 Conduct health education work and conduct health talks (health education) at the village/neighborhood committee; 4 Conduct chronic disease 
management, and issue 2 to 4 weeks of commonly used chronic disease medication to those with stable conditions, and follow-up at regular intervals ( (Chronic Disease Management) 5 Conduct hotline counseling and receive telephone health consultations (hotline consultation) 6 Conduct follow-up telephone calls, call general practitioners to find out (telephone follow-up), 7 Carry out on-site service, fix fixed time for people with reduced mobility On-site service (site service) 8 Conduct rural visits, take the initiative to visit residents' groups for follow-up at a fixed time (rural visits); 9 Open family beds, and establish family beds (family beds) for those who are in demand and who meet the criteria for building beds; 10 Carry out home soothing beds, such as cancer dying patients, regularly Door service (soothing palliative care); Chuanshi to carry out two-way referral, referral work step by step, referral (two-way referral) is indeed superior to the hospital's needs. The scores for each item are 0 to 10 points. 0 points indicate that the job is meaningless, points indicate that the job is optional, 5 points indicate that the work is of general significance, 8 points indicate that the work is more meaningful, and 10 points indicate that the work is very meaningful. (3) Satisfaction of the family doctor responsibility system includes only one item: Satisfaction (satisfaction) of the general work situation of the large group rural health service center in the previous stage. The entry scored 0 to 10 points, 0 being very unsatisfactory, and 10 being very satisfied. Cronbach's $\alpha$ of the questionnaire was 0.839.1.3 Survey Method Prior to the start of the survey, investigators trained investigators to describe the purpose and methods of the survey, and to interpret the questionnaire at the time of the survey. In February-March 2015, the residents were notified by phone to the village clinic or the rural health service center to receive a questionnaire survey. The contracted residents who came to be surveyed explained the purpose of the survey in detail and signed the informed consent after obtaining the consent. , Then conduct a face-to-face investigation. All questionnaires were collected and inspected on the spot. If there are any missing items, they should be filled on the spot. A total of 1100 questionnaires were distributed, 1046 were recovered, 1033 valid questionnaires were recovered, and the effective recovery rate was 93.9\%.

SPSS 20. 0 statistical software was used for data entry and statistical analysis. The 计数 2 test was used for the count data, and the multivariate Logistic regression analysis was used for the influencing factors analysis. The difference was statistically significant at $\mathrm{P}<0.05$.

\section{Results Analysis}

Among the 1,033 contracted residents, 469 were men (45.4\%), 564 were women (54.6\%), and 18-92 years old (60. $5 \pm 11.7$ ) years old, including < 45 The age of 93 cases (accounting for 9.0\%), 45 to 59 years old 332 cases $(32.1 \%), \geq 60$ years old 608 cases $(58.9 \%)$; the education level is elementary school and below 442 cases (accounting for 42. 8\%), 432 cases (41.8\%) in junior high school, 159 cases (15.4\%) in high school/secondary school and above, 814 cases (78.8\%) in occupations, and 219 cases (21) 2\%); Insured type is 831 cases of new rural cooperative medical insurance (accounting for 80.4\%), 202 cases of other medical insurance (accounting for 19.6\%), and 659 cases of chronic diseases (accounting for 63.8\%). There were 374 cases of chronic diseases (accounting for $36.2 \%$ ).

Of the 1,033 contracted residents, 350 (33.9\%) said that "the basic medical treatment in rural areas was fixed at the contracted doctors", 491 cases (47.5\%) said that "the basic medical services in rural areas are not fixed, and sometimes Signing doctors and sometimes visiting other doctors", 192 cases (accounting for 18.6\%) said that "the basic medical service in rural areas does not go to the doctor's office"; 388 cases (accounting for 37.6\%) said that "a contracted family doctor can To solve the problem of basic medical services in rural areas, 570 cases (accounting for 55.2\%) said that "signing family doctors can solve some rural basic medical services problems", 75 cases (accounting for 7.2\%) said that "signing family doctors cannot solve rural basic problems" Medical service issues."

The contracted residents scored 7. $68 \pm$ of 11 service items for rural clinics, appointment clinics, health education, chronic disease management, hotline consultation, telephone follow-up, on-site service, rural visits, family beds, soothing care, and two-way referral. 2. 25), (7. $67 \pm 1.97),(7.64$ 


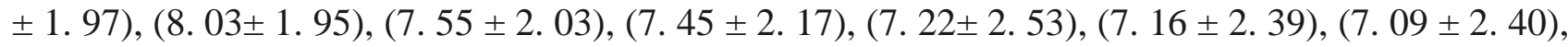
(7. 18 \pm 2. 39), (8. $13 \pm 1.95)$ scores were all over 7 points, among which the two-way referral, chronic disease management, and rural diagnosis and treatment service scores ranked in the top three, and the family beds, rural visits, and soothing care services ranked the last three. The satisfaction score was $(7.89 \pm 1.89)$ cents.

Analysis of the Influencing Factors of the Effect and Satisfaction of the Family Doctor's Responsibility System This study assigned a score of $\geq 8$ points for each service item's effect and satisfaction rating to 1,0 for a score of 0 , and conducted single-factor and multi-factor analysis of the influencing factors.

The results of univariate analysis showed that the residents of different ages had statistically significant differences in appointment clinics, hotline consultations, telephone follow-ups, two-way referrals, and satisfaction ratings $(\mathrm{P}<0.05)$; contracted residents of different educational levels There was a statistically significant difference in the evaluation of soothing treatment and two-way referral $(\mathrm{P}<0.05)$. There was a statistically significant difference in the evaluation of rural clinics and appointment clinics among contracted residents of different occupations $(\mathrm{P}<0.05)$; Different health care contracted residents have a statistically significant difference in the evaluation of chronic disease management $(\mathrm{P}<0.05)$; There are no contracted residents with chronic diseases to rural clinics, appointment clinics, health education, hotline consultation, rural Visits, soothing treatments, two-way referrals, and satisfaction evaluations were statistically significant.

\section{Discussion}

Signed residents have low effective utilization of family doctor's responsibility system services. Family doctor responsibility system services are based on the signing of family doctors and residents and the establishment of a fixed medical treatment relationship [2]. Dadun Rural Health Service Center adopted the " $3+1.5+0.5$ " service mode, ie, 3 days of daily routine work of the center, 1.5 days of sinking rural medical care services, and 0.5 days of center appointment clinics. Only $33.9 \%$ of contracted residents and family doctors have established a fixed relationship between clinicians. Signed residents have low effective utilization of family doctor signing services. The relationship between family doctors and contracted residents has not yet been established. The main reasons for the analysis are: (1) There is no fundamental change in the residents' own mode of thinking for visiting doctors, and they are reluctant to use appointment clinic services; (2) Most family doctors work in wards, only 0.5 days per week at the center to make appointments for outpatient visits. The time is short and the visiting time cannot be fixed. (3) Family doctors are not enthusiastic about fixed diagnosis and treatment, and the fee mechanism has not been linked to the family doctor's interest. The introduction of minor illnesses into rural areas and serious illnesses into hospitals is an important goal of China's reform of the medical and health system. It is also an important means to solve the problem of "difficulties in seeing a doctor and expensive medical treatment”. Can a family doctor's comprehensive service ability meet the medical service needs of contracted residents? Among the key issues [3], this survey shows that only $37.6 \%$ of contracted residents believe that family doctors can solve the common medical problems of rural residents. The reasons for the analysis are: (1) There are 42 general practitioners in the center, There are only 3 general practitioners (7.1\%) who have standardized physician training, and 39 other specialists have been transferred from post-training training. The overall service capacity is not strong; (2) Existing family doctors mostly use clinical diagnosis and treatment as Mainly, public health work is less involved, and it has never been contacted before. The family doctor has "general knowledge, health care, medical treatment, rehabilitation, and other systems of general medical knowledge, and implements comprehensive, continuous, timely and personalized services for the clients in the jurisdiction. There is still a large gap between the requirements of the healthcare services and care [4]; (3) The mechanism of keeping costs has not yet been established, and the family doctors Public health services are not enthusiastic.

The responsibility of the family doctors' responsibility system in the DaChu Rural Health Service Center was carried out since 2010. By the end of 2014, there were a total of 58,661 
residents signed up, 205,663 visits to chronic diseases, 28,537 appointments, 13,292 visits, and hotline consultations. There were 14357 person-times and 1,361 health education activities. [5] The survey shows that the contracted residents have a higher degree of satisfaction with the service content of the family doctor responsibility system, which is consistent with the findings of Liu Deqi et al. Among them, contracted residents generally believe that it is meaningful to carry out two-way referral, chronic disease management, and rural medical treatment; the assessment of family beds, rural visits, and soothing care is relatively low, and the next step is to improve basic general service programs to improve service efficiency and services. value.

\section{Conclusion}

In order to understand the current status of family doctor signing services in rural areas and analyze existing problems, interviews were conducted among the six township and township officials in Ya'an City, Sichuan Province. The results showed that the current family doctor signing services had insufficient primary health care services and contracted service packages. The lack of scientific design, lack of assessment and incentive mechanisms, inadequate equipment and basic medicines, and difficulties in information construction. To this end, it is necessary to improve grassroots service capabilities, clarify the connotation of contracted services, establish assessment incentive mechanisms, improve family doctors' enthusiasm for work, equip and improve medical equipment, expand the scope of grass-roots drug use, build regional information sharing platforms and county medical communities, and promote family doctor signing services.

\section{Acknowledgements}

The Shaanxi Provincial Education Department special scientific research. Project: Performance evaluation of rural family doctor service mode based on Donabedian model in southern Shaanxi Province Project number:17JK0193, Project Leader: Lili Jia. The humanities and social sciences of the Ministry of Education research project: Evaluation of the implementation of family doctor system in western urban communities Project number:15YJA630081, Project Leader: Qingxiang Xue The discipline of public administration in Shaanxi University of Chinese Medicine Project number:500102-138070006, Project Leader: Xiuqin Li

\section{References}

[1] Zhou Yuan, Jiang Qicheng. Current status and problems of family doctor service research in the context of China's grading diagnosis and treatment[J]. Chinese Journal of Rural Health Care, 2017, 37(6):633- 634.

[2] Xue Jinhua. Difficulties and Countermeasures in Implementing the Service Model of Rural Family Doctors' Responsibility System[J]. Chinese contemporary medicine,2011,18(11):125- 126.

[3] Shen Shuguang, Zhang Bo. Graded diagnosis and treatment, first-level primary diagnosis and primary health care institution construction [J]. Xue Hai, 2016, 4(2):48-57.

[4] Pan Xuejun, Wang Zhengping. Practice and thinking of family responsibility system in rural areas in the outer suburbs [J]. Medical aesthetics, 2014, 23 (12): 568.

[5] Gao Maolong, Wang Jing, Wang Jintang, et al. The awareness rate and its influencing factors of rural elderly homelessness in Beijing [J]. Chinese General Practice, 2014, 17 (19): 2262- 2264. 